\title{
Male brain theory of autism
}

The "extreme male brain" theory of autism was initially proposed in 2002 by Professor Simon Baron-Cohen, who founded the Autism Research Centre in Cambridge, UK. Autism spectrum disorders (ASD) have always been more commonly diagnosed in males than females, with a ratio of four boys to every girl. Hans Asperger, whose name was later used to describe highly verbal autistic people, had never seen a female patient. He made the prescient suggestion that "Even within the normal variation, we find typical sex differences [in intelligence] ... in the autistic individual, the male pattern is exaggerated to the extreme".

The extreme male brain theory of autism reformulates Asperger's comment not along the lines of intelligence, but instead with respect to two psychological factors. This theory places people with autism on the far end of a spectrum that extends into the typical population with respect to empathy and systemizing. This article first describes these two factors and then considers later developments to the theory and finally its critical reception by scientists.

\section{Empathizing and systemizing}

Empathizing is the common human drive to share another's affective (emotional) state. It is, however, actually a little more complicated, and involves a cognitive as well as an affective component. The cognitive aspect of empathy is also known as 'theory of mind' or 'mentalising': the ability to attribute beliefs and mental states to other people based on their behaviour. The affective aspect of empathy is the emotional response that the individual feels in accordance with their understanding of the other person's mental state. If Sally sees Anne crying, she infers from her behaviour that Anne is sad (cognitive empathy), and might go further to guess the reason from prior knowledge: maybe Anne believes she has lost her job? The affective component of empathy is the sadness that Sally feels on observing her friend's sadness; the same process of mirroring another's emotions might occur when Sally observes Anne's joy and feels happy herself.

Systemizing is the drive to find patterns or rules in seemingly arbitrary data. Systems are all around us: there are obvious systems in technology (a computer and programming) or language (the rules of syntax), but there are also less obvious systems, for example, in the natural world (the weather, the phases of the moon), social systems (politics, business hierarchies), and in behaviour (techniques that one adopts for playing a sport or a musical instrument). Systemizing involves scrutinising data in immense detail so that one can uncover laws that will allow one to predict future events, behaviour or data as it fits within a system. Systemizing also includes the drive to create systems in one's own life, for example by carefully sorting and categorising items.

These independent drives to empathise and systemize are present in all of us to varying degrees and in some cases are well-balanced, but Baron-Cohen noticed a trend where men tend to be higher in systemizing than empathizing ("type $S$ ", or $S>E$ ) and women tend to be higher in empathizing than systemizing ("type E", or E $>$ S). Among other expressions of 
empathy, he pointed out that girls and women tend to show more concern for fairness and turn-taking; are less aggressive, less likely to engage in rough play and more likely to comfort others; show more concern for and affective response to the emotions of others, and are accordingly more sensitive to nonverbal communication, tone of voice and facial expression. In contrast, boys prefer mechanical, 'systemizable' toys like vehicles and building blocks, and males perform better at certain tasks involving systemizing. Certain disciplines like maths, physics and engineering are heavily male-dominated, as are occupations like metalworking, weapon making and construction. Caring professions, in contrast, have been historically female-dominated.

If we view systemizing and empathizing on two dimensions, the typical population might look something like this:

Of course, there are men who are higher in empathizing than systemizing, women who are higher in systemizing than empathizing, and people who are higher or lower in both. However, the theory suggests a population-level trend where most females are higher in empathizing than systemizing and most men show the opposite pattern.

With respect to empathizing and systemizing, Baron-Cohen suggested that males and females with autism ('A' in the figure) show an extreme form of this male-trend: they struggle to emphasize whilst showing a hyperactive drive to systemize. Autistic individuals, described elsewhere by Baron-Cohen as 'mindblind', appear to have impaired or delayed development of the cognitive aspect of empathy. A common misunderstanding is that people with autism lack feeling (the affective component), but in fact they struggle to first make sense of behaviour in terms of understanding the thoughts and feelings of others. In contrast, their high drive to systemize is reflected in their intense attention to detail; their preference for factual, rule-based information; their tendency to collect and organise items, and preference for constructional toys; their preference for sameness and routine; and their inclination towards predictable systems, like computers. Some individuals with autism even display remarkable skills in systemisable domains like maths, calendrical calculation, syntax acquisition, music, or memory for circumscribed types of information.

The anxiety and difficulty that people with autism experience in social interactions is explained by the nature of human behaviour and the social world, which defies systemizing. Childhood games, friendships, professional and romantic relationships and the social niceties that govern everyday life are highly unpredictable and unstructured. Some people with autism are able to learn scripts or rules for social interaction, but as these are rather mechanical, dependent on their systemizing ability rather than innate capacity for cognitive and emotional empathy, they lack the finesse to adapt quickly to the complex social world.

Autism varies in severity, but the extreme male brain theory suggests that all autistic individuals share the " $\mathrm{S}>\mathrm{E}$ " profile of hyper-systemizing, hypo-empathizing. This would however emerge very differently depending on the individual. Systemizing in somebody with Asperger's Syndrome might express itself as a meticulous knowledge of different breeds of dogs, collecting facts about their life expectancies, typical measurements, preferred foods and 
so forth. An individual with classic, non-verbal autism might instead line objects up, or be immensely preoccupied with their familiar placement in a room, such that minute changes in organisation, like changes in known routines, cause major distress.

In 2014, Baron-Cohen and colleagues conducted an internet survey of nearly 5000 adults. They found that both males and females with autism showed a shift towards the typical male profile $(S>E)$, and that there were less obvious differences in empathizing and systemizing between males and females with autism than between typically-developed males and females.

\section{Later developments: brain and hormones}

Although the extreme male brain theory of autism was originally based on male and female trends in empathizing and systemizing, later research extended this cognitive theory to neuroanatomy. One study found that brain areas that are smaller in males tend to be even smaller in people with autism, and brain areas that are larger in males are even bigger in people with autism. ASD are extremely heterogeneous, however, and this pattern has not always been replicated.

In 2011, Baron-Cohen and colleagues suggested a link between hyper-masculinisation of behaviour and prenatal exposure to androgens. Developing foetuses are exposed to substantially greater levels of testosterone if they are male. Testosterone not only causes the differentiation of the genitalia but masculinises the structure of the brain. Accordingly, prenatal testosterone exposure also impacts on behaviour: rate of language development, social interaction, eye contact and empathy decrease with testosterone exposure, whilst systemizing and attention to detail increase.

What about people with autism? In accordance with the above, the extreme male brain theory might suggest that these individuals may experience abnormally high exposure to testosterone at a critical period of prenatal development. Infants with greater prenatal exposure to testosterone were indeed shown to have significantly more autistic traits. Furthermore, autistic women and their mothers have greater than normal rates of testosterone-related medical conditions, like hirsutism, acne, and polycystic ovary syndrome, suggesting current testosterone abnormalities. Investigation of the role of sex hormones in ASD is still ongoing.

\section{Critical response to the extreme male brain theory}

Whilst some studies support the predictions of extreme male brain theory, it is not universally supported. Some of the evidence cited in favour of the theory, such as the preference of male children for construction toys and their superiority in tasks involving systemizing, presumes that these features are innate and ignores the effects of culture and socialisation on development. Female strengths in empathizing may also be related to nurture, not nature. It 
may be that people with autism are less affected by societal pressures, but the original theory and its later link to testosterone does not consider any role of social conditioning.

There certainly appear to be sex differences in empathizing and systemizing, the measures that Baron-Cohen created, and people with autism appear to show weaknesses in the former and strengths in the latter. However, other theorists have called for a closer look at the relationship between autism and 'systemizing' and 'empathizing' as they might occur in behaviour. The link between foetal testosterone and actual diagnosed cases of autism remains to be demonstrated.

On broader grounds, there is also concern that the extreme male brain theory might inadvertently contribute to under-diagnosis of females with autism. This is already a problem with potentially serious repercussions, such as increased risk of psychiatric illnesses. The later linkage of the theory to foetal testosterone suggests that the gender bias in diagnosis is a real one, since male foetuses are undoubtedly exposed to greater quantities of testosterone. However, others have argued that the gender imbalance in autism diagnosis may be an artefact, resulting from the use of biased diagnostic instruments which were developed from male observations. In this view, it would be important to recognise that perhaps just as many girls have autism, but may express it differently.

Rachel Louise Moseley

\section{See also:}

Empathy, theory of mind, Asperger's Syndrome, gender differences, autism spectrum disorders (biology of), hormones.

\section{Further reading:}

Auyeung, B., Taylor, K., Hackett, G., \& Baron-Cohen, S. (2010). Foetal testosterone and autistic traits in 18 to 24-month-old children. Molecular Autism, 1(11).

Baron-Cohen, S. (2002). The extreme male brain theory of autism. Trends in Cognitive Sciences, 6(6), 248-254.

Baron-Cohen, S. (2009). Autism: the empathizing-systemizing (E-S) theory. Annals of the New York Academy of Sciences, 1156(1), 68-80.

Baron-Cohen, S., Cassidy, S., Auyeung, B., Allison, C., Achoukhi, M., Robertson, S., ... \& Lai, M. C. (2014). Attenuation of typical sex differences in 800 adults with autism vs. 3,900 controls. PLOS One, 9(7), e102251. 
Baron-Cohen, S., Knickmeyer, R. C., \& Belmonte, M. K. (2005). Sex differences in the brain: implications for explaining autism. Science, 310(5749), 819-823.

Baron-Cohen, S., Lombardo, M. V., Auyeung, B., Ashwin, E., Chakrabarti, B., \& Knickmeyer, R. (2011). Why are autism spectrum conditions more prevalent in males. PLoS Biology, 9(6), e1001081.

Gould, J., \& Ashton-Smith, J. (2011). Missed diagnosis or misdiagnosis? Girls and women on the autism spectrum. Good Autism Practice (GAP), 12(1), 34-41.

Ingudomnukul, E., Baron-Cohen, S., Wheelwright, S., \& Knickmeyer, R. (2007). Elevated rates of testosterone-related disorders in women with autism spectrum conditions. Hormones and Behavior, 51(5), 597-604.

Krahn, T. M., \& Fenton, A. (2012). The extreme male brain theory of autism and the potential adverse effects for boys and girls with autism. Journal of Bioethical Inquiry, 9(1), 93-103. 